UDC548.75, 538.91

\title{
Methane in water cryomatrix-physical modeling of the formation of the clathrate hydrates of methane
}

\author{
Drobyshev A., Aldiyarov A., Kurnosov V., Korshikov E., Sokolov D., Timchenko A., \\ Shinbayeva A., Katpaeva K. \\ Al-Farabi Kazakh National University, al-Farabi71,050040 Almaty, Kazakhstan
}

\begin{abstract}
Nowadays natural gas hydrates attract special attention as a possible source of fossil fuel. According to various estimates, the reserves of hydrocarbons in hydrates exceed considerably explored reserves of natural gas. Due to the clathrate structure the unit volume of the gas hydrate can contain up to 160-180 volumes of pure gas. In recent years interest to a problem of gas hydrates has considerably increased. Such changes are connected with the progress of searches of alternative sources of hydrocarbonic raw materials in countries that do not possess the resources of energy carriers. Thus gas hydrates are nonconventional sources of the hydrcarbonic raw materials which can be developed in the near future.

At the same time, mechanisms of methane clathrate hydrates formations have not reached an advanced level, their thermophysical and mechanical properties have not been investigated profoundly [1]. Regarding this experimental modeling of the processes of formational clathrate hydrates of methane in water cryomatrix in the process of co-condensation from gas phase on cooled substrate was carried in the range of condensation temperatures $\mathrm{T}=(12-60) \mathrm{K}$ and pressures $\mathrm{P}=\left(10^{-4}-10^{-6}\right)$ Torr. In this experiment concentration of methane in water varies in the range of $1-10 \%$. The thickness of a film was $30-60 \mathrm{mcm}$. The vibrational spectra of twocomponent thin films of cryovacuum condensates of $\mathrm{CH}_{4}+\mathrm{H}_{2} \mathrm{O}$ were measured and analyzed.
\end{abstract}

Keywords: methane, gas hydrates, cryomatrix-physical modeling, clathrate hydrates formations.

PACS number(s): 61.50.-f, 68.35.Rh

\section{Introduction}

Interest to studying hydrates of methane is caused both the applied importance of such researches, and fundamental interest. When we speak about practical application of the knowledge connected with processes of formation and properties of clathrates of methane, first of all that we have to know the main deposits of methane are concentrated on bottom of the World Ocean and exist in the form of clathrates. The solution of the problem of production of these minerals is an important scientific and applied task.

Beside, there are numbers of questions, which are connected with interaction of the molecules methane with other substances, having a greater extent heuristic meaning. In particular, it is a question of interaction in water molecules when quantum features of methane, such as closed interrelation of oscillatory and rotary modes of a molecule is fully shown. The second circumstance is connected with properties of molecules of water to form grids of

* Corresponding author: e-mail: Drobyshev@kaznu.kz hydrogen communications. The combination of these properties leads to formation of the systems consisting of molecules of methane, surrounded with a grid of the molecules which are connected with hydrogen atoms - methane clathrates.

The real research is devoted to studying of such systems. Thus feature of our researches is the following. We suppose that the behavior of methane in the water environment with high pressures (as it is carried out at a depth of 2-3 km) can quite be modeled by a method of cryomatrix isolation at big concentration. We suppose that interposition of a methane molecule in a lattice of ice of water can be similar as an environment of clathrates existence. Ice can create the same static influence, as well as water at a great depth. In this case we can simply obtain the studied object and conduct it's researches in vitro.

Vibrational ranges of two-component mixes (water and methane), formed in various conditions, lie at the heart of a technique of formed films studying. Detected features can be interpreted as existence of the connected states of methane in solid solution with water. Thus the co-condensation method from gas mix on cooled substrate gives oppor- 
tunities to change over a wide range experimental conditions, and also to study thermostimulated processes in objects.

\section{Experiments and measurement procedure}

At the core of procedure for obtaining information about the state of methane molecules in the matrix of different gases is an analysis of the absorptive amplitude of band, which corresponds to the vibrations of the methane molecule in the unbounded state. The measurements were carried at the system, which scheme is given in Picture.1. The main unit of the system is a cylindrical vacuum chamber (1) in diameter and a height of $450 \mathrm{~mm}$. Pumping out the vacuum chamber was carried out by turbo-molecular pump Turbo-V-301 (2), which was connected to the chamber through the sliding vane gate valve CFF-100 (3). As a backing vacuum pump dry spiral pump SH-110 (not shown at the picture) was used. The ultimate vacuum in the chamber reaches the value not worse than $\mathrm{P}=10^{-8}$ Torr. Measuring the pressure in the chamber was carried out with wide-range pressure transducer FRG-700 (4) with the controller AGC-100.

In the center of the chamber the microcryogenic system of Gifford-McMahon (5) is located. On the top flange of microcryogenic system a mirror substrate (6) serving as condensation surface of mixture of methane and water is mounted. The substrate is made from copper, the working surface of which is covered with silver. The diameter of the substrate is $\mathrm{d}=60 \mathrm{~mm}$. The minimum temperature of condensation is $\mathrm{T}=12 \mathrm{~K}$. The temperature measurement was carried out by silicon sensor TS 670-1.4 using a temperature controller M335/20c. Measurement of thickness and rate of condensation is carried out by a double-beam laser interferometer based on photoelectron-multiplier P25a-SS-0-100 (7). IR absorption spectra was measured in the frequency range $400 \mathrm{~cm}^{-1}-4200 \mathrm{~cm}^{-1}$.

To obtain a mixture of the test substance with a matrix gas calibrated volume (not shown at the picture) was used. At first the scope was filled up to necessary pressure with the investigated gas (methane). Typically, the pressure value was 1-1,5Torr. Thereafter, the calibration volume was filled up to the required pressure with water vapor, which corresponds to a working concentration. For the preparation of the mixture the pressure controller PR 4000 (MKS) was used with an accuracy of measurement of pressure 0,01 Torr.

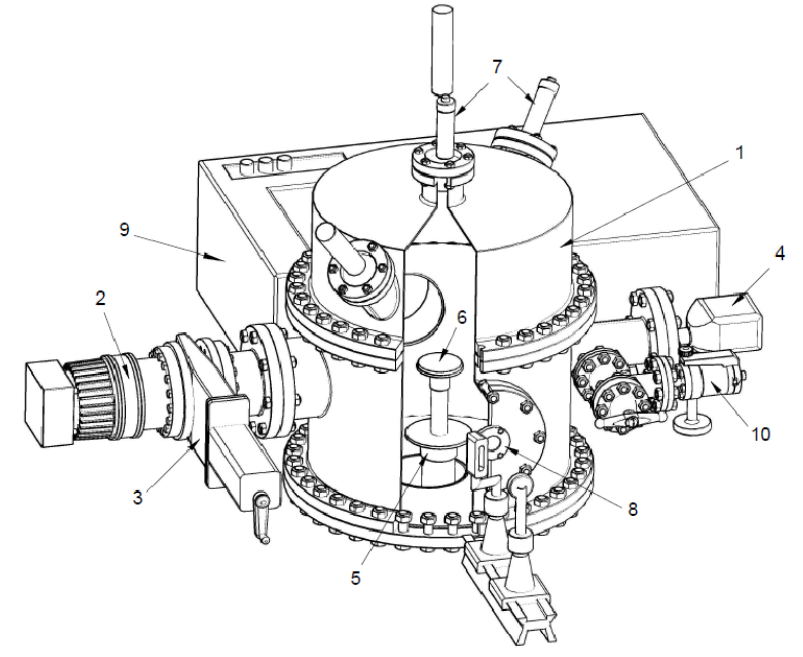

Figure 1 - Experimental installation. 1 - vacuum chamber; 2 - pump Turbo-V-301, 3 - sliding vane gate valve CFF-100; 4 -- pressure transducer FRG-700; 5 - microcryogenic system of Gifford-McMahon; 6- substrate; 7

- photomultiplier; 8 - optical channel; 9 - IRspectrometr; 10 - lake system.

There is a following procedure for the performance of the experiment. The vacuum chamber was pumped out up to a pressure of $\mathrm{P}=10^{-8}$ Torr, then to prevent contamination the substrate was overlapping with the protective plate and its cooling up to $\mathrm{T}=12 \mathrm{~K}$ was carried out. With the leakage system (10) in the chamber the operating pressure of the mixture $\mathrm{P}=10^{-5}$ Torr was setting, the substrate was opening and the process of film cryocondensation begun, it was controlled by double-beam laser interferometer. Once the sample thickness reached of about 25-30 $\mu \mathrm{m}$ gas filling was stopped and a pressure in the chamber was set about $\mathrm{P}=10^{-8}$ Torr. Next, the vibrational spectrum of the sample was measured, whereupon IR spectrometer was installed at a frequency of observation and an interferometer signal was measured at a constant temperature equal to the condensation temperature $\mathrm{T}=16 \mathrm{~K}$ within 30 40 minutes. Thus, the state of the sample was analyzed over time at a constant temperature.

Further measurements were carried out by two methods. In one case, was carried out the step heating of the sample by $0.5-1$ degree with the measurement of the reflectance spectrum at a fixed temperature. In the second case, was carried out the continuous heating of the sample, the speed of which determined by the natural heat inflows to the substrate with switched off microcryogenic machine. In this case, the IR-spectrometer signal was measured at a fixed frequency in the vicinity of the characteristic vibration frequencies of the water mo- 
lecule. Change in a given signal is a reflection of transformations in the test sample.

\section{Results and discussion}

In the present study we used methane gas produced by company IHSAN TECHNOGAZ. Gas purity was $99.99 \%$. The picture. 2 shows the vibration spectrum of methane in gas state (bottom spectrum) and solid state (upper spectrum). Spectrum of methane in gas state was measured at a pressure $\mathrm{P}=$ 4 Torr and a temperature $\mathrm{T}=300 \mathrm{~K}$. The solid sample was obtained in the course of methane cryovacum condensation of gas at a pressure $\mathrm{P}_{\mathrm{c}}=10^{-5}$ Torr at a substrate temperature is maintained at $\mathrm{T}=16 \mathrm{~K}$. The thickness of the sample was $5 \mathrm{mcm}$. We will not go into a detailed analysis of those spectrums, bearing in mind that these measurements have predominantly gauge character. We only note that cryocondensation of methane leads to freezing of the rotational subsystem of methane, resulting in a transformation of the gas spectrum in two fairly narrow absorption bands. The maximum absorption band of $\mathrm{C}-\mathrm{H}$ stretching vibrations is located at the frequency of $v=3016 \mathrm{~cm}^{-1}$. Corresponding band of deformation vibrations has a minimum at a frequency of $v=1306 \mathrm{~cm}^{-1}$. These data is needed for analyzing the state of the methane molecule in the solid solution in water.

The process of formation of methane hydrates as sumes the existence of certain features in the interaction of methane molecules with a lattice of ice water. To do correct assessment of this effect it seems appropriate to investigate the effect of neutral cryomatrix on the vibrational spectra of methane. In addition, these measurements are not the main purposes of these studies, and they have a calibration character.

Picture. 3 shows the results of these studies in a $5 \%$ solution of methane with nitrogen and argon. The picture. 3 (a) shows the data in the frequency range of $\mathrm{C}-\mathrm{H}$ stretching vibrations, and the figure on the right shows the corresponding data for deformation vibrations. These are compared with the corresponding absorption bands of cryocondensed pure methane. The samples shown in picture. 3 were condensed at a substrate temperature $\mathrm{T}=16 \mathrm{~K}$ and a pressure of the gas phase $\mathrm{P}=10^{-5}$ Torr. The sample thickness was $\mathrm{d}=10 \mathrm{mcm}$.

As seen in picture.3, the absorption band of the stretching and deformation vibrations of methane molecules in solution with argon and nitrogen markedly shifted to higher frequencies relating to pure methane, this is a result of the influence which lattice of matrix has on the methane molecules. Interestingly, the amount of offset of the valence band is almost the same as the fluctuations of the methane- nitrogen mixtures and mixtures of argonmethane and the value is about $9 \mathrm{~cm}^{-1}$. At the same time, deformation of the absorption band of oscillation (pic.3(b)) of a mixture of methane and argon is shifted to by $6 \mathrm{~cm}^{-1}$, and the methane- nitrogen mixture by $9 \mathrm{~cm}^{-1}$.

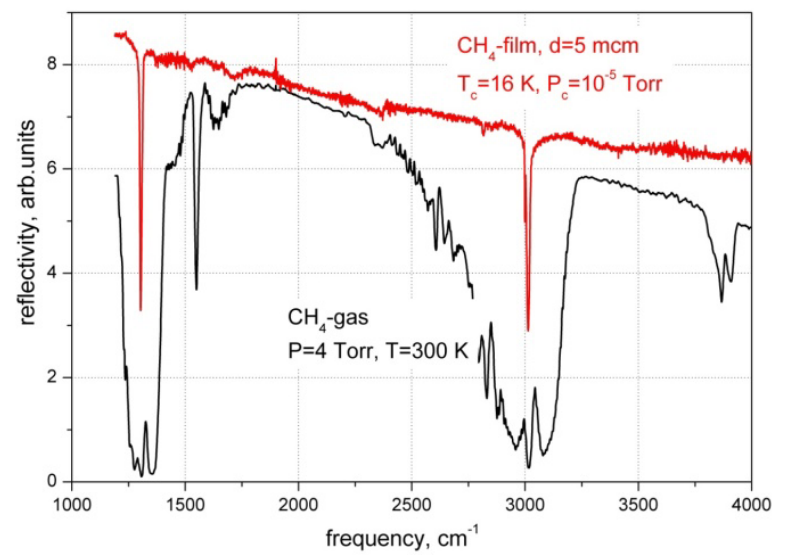

Figure 2 -The rotational-vibrational spectrum of methane in gas and solid state.

In the present article we present the results of studying properties of thin films of cryocondensates of two-component mixes of methane and water in the range of concentration ratios from $5 \%$ to $90 \%$. The general scheme of carrying out experiments included the following stages. Mixture of gaseous methane and vapors of water of the specific concentration was filled in the vacuum camera which was previously pumped-out up to the pressure of $\mathrm{P}=10^{-8}$ Torr and condensed on a substrate at a temperature of $\mathrm{T}=16 \mathrm{~K}$ and pressure of $\mathrm{P}=10^{-5}$ Torr. Sample thickness usually was $10-15$ $\mathrm{mcm}$. It is important to note that due to the change of concentration of mixture film thickness also varied so that the effective mass of methane in various samples remained approximately same. Measurement of an oscillatory range in the range of characteristic frequencies of fluctuations of a molecule of methane was carried out on reaching the set parameters of a film. Further measurements were carried out according to two schemes which have been used by us in earlier investigations.[2, 3]In the first case, ranges were taken at the fixed values substrate temperatures in the course of its increase. Thus the behavior of strips of the 
absorption of methane was controlled. In the second technique, the frequency of an IRspectrometer was set on value of the any maximum absorption strip of methane. As a rule, we set the frequency of deformation fluctuations because, in difference from valent $\mathrm{CH}$-fluctuations, this frequency concerning strip is separated from stripes of absorption of water. Further we measured the absorption amplitude at a chosen frequency in the course of slow temperature increase of a substrate before the sample evaporated. The obtained thermograms continuously reflecteda condition of samples in the course of their heating.

In the present article we present the results of studying properties of thin films of cryocondensates of two-component mixes of methane and water in the range of concentration ratios from $5 \%$ to $90 \%$. The general scheme of carrying out experiments included the following stages. Mixture of gaseous methane and vapors of water of the specific concentration was filled in the vacuum camera which was previously pumped-out up to the pressure of $\mathrm{P}=10^{-8}$ Torr and condensed on a substrate at a temperature of $\mathrm{T}=16 \mathrm{~K}$ and pressure of $\mathrm{P}=10^{-5}$ Torr. Sample thickness usually was $10-15$ $\mathrm{mcm}$. It is important to note that due to the change of concentration of mixture film thickness also varied so that the effective mass of methane in various samples remained approximately same. Measurement of an oscillatory range in the range of characteristic frequencies of fluctuations of a molecule of methane was carried out on reaching the set parameters of a film. Further measurements were carried out according to two schemes which have been used by us in earlier investigations.[2, 3]In the first case, ranges were taken at the fixed values substrate temperatures in the course of its increase. Thus the behavior of strips of the absorption of methane was controlled. In the second technique, the frequency of an IRspectrometer was set on value of the any maximum absorption strip of methane. As a rule, we set the frequency of deformation fluctuations because, in difference from valent $\mathrm{CH}$-fluctuations, this frequency concerning strip is separated from stripes of absorption of water. Further we measured the absorption amplitude at a chosen frequency in the course of slow temperature increase of a substrate before the sample evaporated. The obtained thermograms continuously reflecteda condition of samples in the course of their heating.

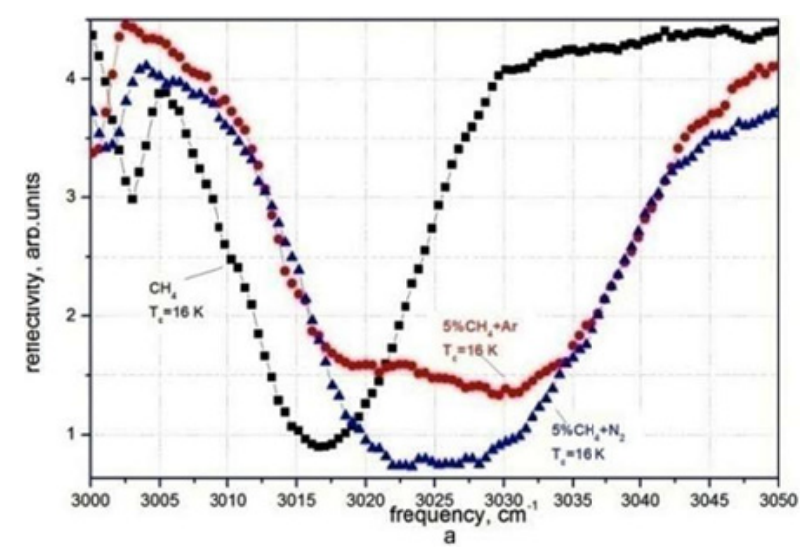

a)

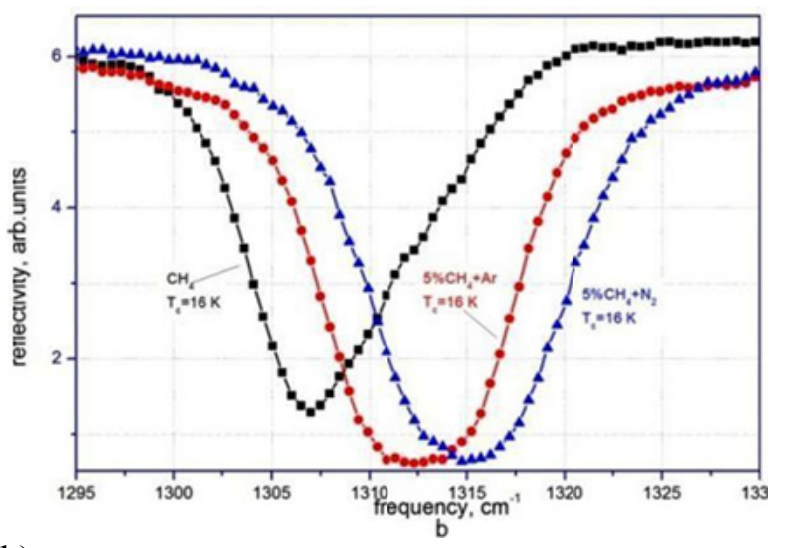

b)

Figure 3 - IR spectrums of thin films of pure methane and its 5\% mixture with nitrogen and argon in the ranges of valence (a) and deformation (b) $\mathrm{CH}$ fluctuation frequencies.

One more important measured parameter in our experiments is change of pressure in the vacuum camera. In the course of its measurement the pumping valve of the camera was slightly opened to pump out the residual gases which are consequences of a inleakage in the camera and change of equilibrium pressure at temperature increase. Thus, spontaneous thermodesorption increase of pressure in the camera may be connected with the processes happening in a sample, which are reflected in the characters of the thermograms which were obtained at measurement of a signal of an IR-spectrometer. This method of thermal desorption was widely used earlier by us and some other authors [4-6]. Thus, measurement of oscillatory ranges of the condensed films and the subsequent analysis of their transformations in the 
course of temperature increase during a methane coheart of physical modeling of processes of formation of hydrates of methane suggested by us.

The vibrational range of the sample consisting of $25 \%$ of methane and $75 \%$ of water is presented in pic. 4. The temperature of the condensation is $\mathrm{T}=16 \mathrm{~K}$, the pressure of the condensation is $\mathrm{P}=10^{-5}$ Torr, the thickness of a film is $\mathrm{d}=10 \mathrm{mcm}$. The main strips of absorption in this spectrum belong to the following fluctuations.

Absorption band with a maximum at a frequency $v=1320 \mathrm{~cm}^{-1}$ corresponds to a deformation (or rotational ) vibrations of $\mathrm{C}-\mathrm{H}$ bond of methane. Wide peak with a maximum at a frequency $v=1676 \mathrm{~cm}^{-1}$ refers to the deformation vibrations of the water molecule. Sharp peak at the specified frequency $v=3020 \mathrm{~cm}^{-1}$ corresponds to the stretching vibrations of $\mathrm{CH}_{4}$, and the following wide peak centered at a frequency $v=3300 \mathrm{~cm}^{-1}$ displays the $\mathrm{OH}$ bond of the water molecule.It is an interesting interpretation of the peak at $v=3670 \mathrm{~cm}^{-}$ ${ }^{1}$. Traditionally fluctuations located in this frequency range are referred to as quasi-free vibrations of monomers or dimers of linear molecules of water in cryomatrix [ 7-9 ]. In the cited works, this peak was a narrow absorption band with a pronounced minimum, whereas in the present studies band at $3670 \mathrm{~cm}^{-1}$ and the deformation peak of water at $1676 \mathrm{~cm}^{-1}$ have a complex fine structure that may be due to the peculiarities of interaction of molecules of water and methane. As seen in Pic. 4, the absorption bands of methane in $25 \%$ mixture of water have little "blue" shift relatively to pure solid methane, amounting to the value of the bending vibration of about $14 \mathrm{~cm}^{-1}$ and for $\mathrm{CH}$ stretching vibrations of approximately $5 \mathrm{~cm}^{-1}$. It is virtually identical to the data for other matrixes (Pic. 2). From this, we can conclude that the state of the methane molecule, its vibrational spectrum is weakly dependent on the composition of the mixtures we discussed in our article.

Infrared spectra of samples condensed at $\mathrm{T}=16$ $\mathrm{K}$ were measured and subsequently warmed with an interval of $2-5 \mathrm{~K}$ until complete evaporation of the sample at a temperature of about $180 \mathrm{~K}$. It was found that up to a temperature of $160 \mathrm{~K}$ the absorption band is presented in the sample corresponding to fluctuations of methane molecule. It is necessary to notice that the values of the equilibrium temperature of methane at operating pressures of the order of $10^{-5}-10^{-6}$ Torr is around $36-38 \mathrm{~K}$, i.e., at substrate temperatures above these values, solid methane condensation with vapors of water lies at the must be completely evaporated, if it is in a free state. We can reliably assume that in this case there may be at least two bound states of methane in water; namely, the methane is adsorbed by cryocondensate layers of water and methane in a state of hydrates. These two states of methane may occur at elevated temperatures in the form of characteristic absorption bands in the IR spectra.

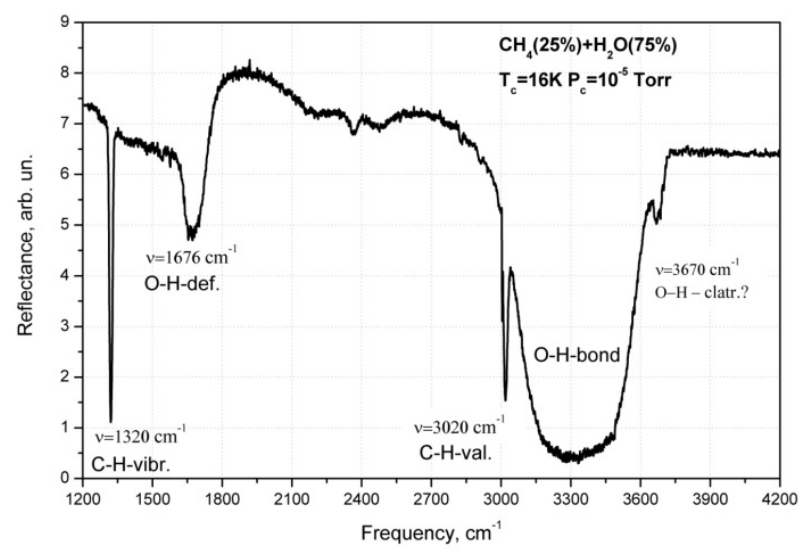

Figure 4 -IR spectrum of the film of cryovacuum condensate of mixture of $25 \%$ methane and $75 \%$ water. Condensing temperature is $\mathrm{T}=16 \mathrm{~K}$; condensing pressure is $\mathrm{P}=10^{-5}$ Torr, film thickness is $\mathrm{d}=10 \mathrm{mcm}$.

As the experience of our previous studies, the observation at a fixed IR spectrometer frequency can give more detailed information about thermally stimulated processes occurring in cryocondensates. As we have said, it is more convenient to observe the behavior of the absorption band at a frequency of deformation vibrations of methane due to its isolation relative to other bands. The results of these measurements are presented in Pic. 5. These thermograms of two concentration ratios of methane and water are obtained by observation at frequency of IR spectrometer $v=1320 \mathrm{~cm}^{-1}$. Thin lines show the corresponding absorption bands, which clearly demonstrate the position of the measured values on the thermograms relatively to absorption bands, i.e. the presence of methane in the sample.

When we're analyzing the thermograms shown in Pic.5, we should note the following basic facts.

1. Methane in the solid solution with water is present in the sample up to the evaporation temperature of water, i.e. to $\mathrm{T}=160-170 \mathrm{~K}$.

2. Character of the thermogram significantly depends on the concentration of methane in water. The small concentration of methane in the gas phase 
leads to the slower and more monotonically changes of the amplitude of absorption of deformation vibrations, and, consequently, the methane content in the sample.

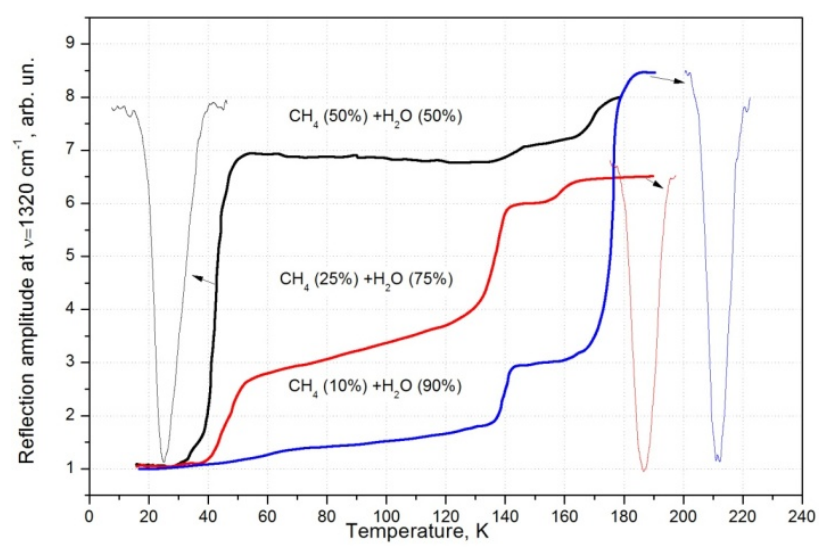

Figure 5 - Thermally stimulated changes in reflectance capacity of samples at the frequency of observation of $v=$ $1320 \mathrm{~cm}^{-1}$. Thermograms are presented with different concentration ratios of methane and water. Thin lines show the absorption bands of the deformation vibrations of methane to the corresponding experiments.

3. Characteristic values of the temperature, which is observed in the region of the most radical changes of the nature of thermograms, are $\mathrm{T}=40 \mathrm{~K}$ (sublimation temperature of pure methane [10]) and $\mathrm{T}=135-140 \mathrm{~K}$ (temperature of the transition of amorphous ice water to state of super cold liquid SCL $[11,12])$.

The comparison of thermograms of the interferometer signal with measurements of pressure in the chamber at the same temperatures significantly complements the information about the processes occurring in the samples when the temperature is rising.

As can be seen from the figures, the character of changes of the amplitude of deformation vibrations of methane molecule depends on the composition of the samples. However, it should be noted that the characteristic of behavior of features of the thermograms for all given concentrations are closely associated with the methane desorption peaks. Thus, the interpretation of the nature of desorption peaks in combination with IR thermograms may hold the key to understanding the processes which are occurring in the samples. In particular, peak 1 in Pic.6 and 7 (at Pic. 8 it is missed), most likely, is not associated with changes in methane concentration in the sample, because his appearance at $\mathrm{T}=20 \mathrm{~K}$ is not accompanied by changes in the amplitude of the de- formation vibrations. Apparently, this peak corresponds to the gas desorption (possibly of nitrogen), which is condensed on the inoperative surfaces of the substrate out of a film. This fact is also mentioned in [13].

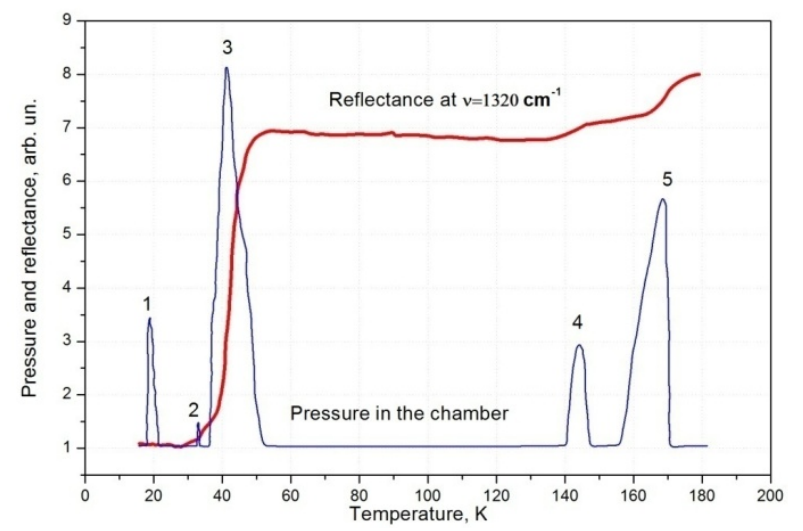

Figure 6 - Change of reflectance of the sample $\left(50 \% \mathrm{CH}_{4}\right.$ $\left.+50 \% \mathrm{H}_{2} \mathrm{O}\right)$ at the frequency of observation $v=1305 \mathrm{~cm}^{-}$

${ }^{1}$ (upper curve) and the pressure in the chamber (bottom curve) in the heating process. Condensation temperature is $T_{c}=16 \mathrm{~K}$, the sample thickness is $\mathrm{d}=10 \mathrm{mcm}$.

Desorption peaks 1 and 2 in Pic. 6, as well as peak 2 in Pic. 7 and peak 1 in Pic. 8 are in full agreement with the change of the amplitude of the absorption frequency of the deformation vibrations of methane $v=1305 \mathrm{~cm}^{-1}$. Start -up of increase of pressure in the vicinity of the sample temperature $\mathrm{T}=40 \mathrm{~K}$ coincides with a sharp decrease of the amplitude of the absorption at the observed frequency of the spectrometer. Further increase of the temperature in the interval from $\mathrm{T}=40 \mathrm{~K}$ to approximately $\mathrm{T}=58 \mathrm{~K}$ is accompanied by increase of the desorption pressure to a maximum value at a temperature $\mathrm{T}=42-43 \mathrm{~K}$ with subsequent reduction to the original value ( $\mathrm{P}=5 * 10^{-7}$ Torr). Maximum values of desorption pressure for the different samples are $\mathrm{P}=2 * 10^{-5}$ Torr in Pic. $6, \mathrm{P}=9 * 10^{-6}$ Torr in Pic. 7 and $\mathrm{P}=4 * 10^{-6}$ Torr in Pic. 8 . When the concentration of methane in the sample is higher, the value of pressure of thermal desorption is increasing.

The obvious presence of fine structure of considered desorption peaks for different samples suggests, that the processes occurring in this temperature range are more complex than just desorption of cryocaptured molecules of methane. However, in this article we are not going to discuss these questions, because of the need more detailed studies of this phenomenon. 


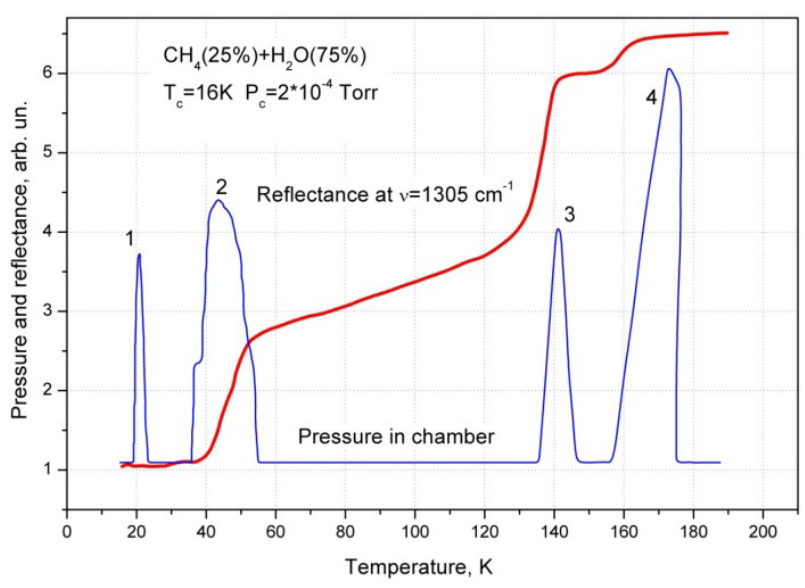

Figure 7 - The change of the reflectance of the sample $\left(25 \% \mathrm{CH}_{4}+75 \% \mathrm{H}_{2} \mathrm{O}\right)$ at a frequency of observation $v=$ $1305 \mathrm{~cm}^{-1}$ (upper curve) and the pressure in the chamber (bottom curve) in the heating process. Condensation temperature is $T_{c}=16 \mathrm{~K}$, the sample thickness is $\mathrm{d}=10$ $\mathrm{mcm}$.

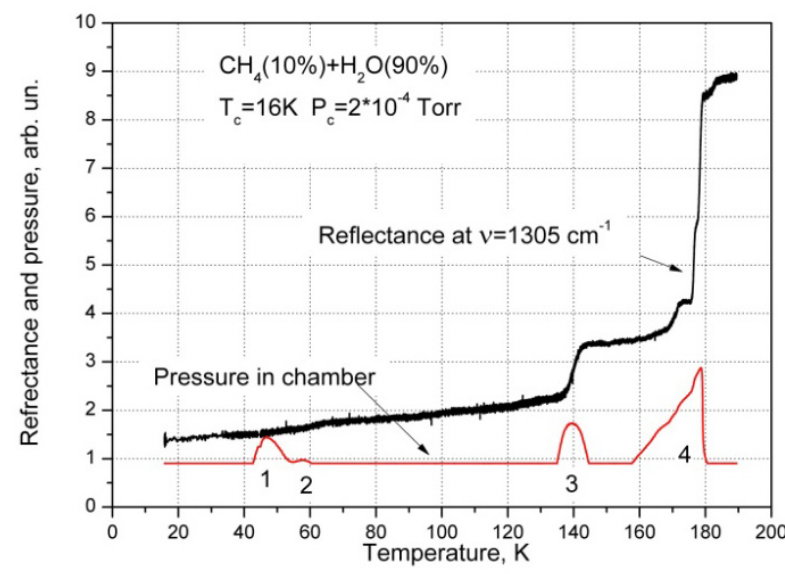

Figure 8 - Change in reflectance of the sample (10\% $\left.\mathrm{CH}_{4}+90 \% \mathrm{H}_{2} \mathrm{O}\right)$ at a frequency of observation $v=1305$ $\mathrm{cm}^{-1}$ (upper curve) and the pressure in the chamber (bottom curve) in the heating process. Condensation temperature is $T_{c}=16 \mathrm{~K}$, the sample thickness is $d=10$ $\mathrm{mcm}$.

Temperature range $145-160 \mathrm{~K}$ is characterized by stabilization processes occurring in all three samples. The pressure in the chamber, and the amplitude of absorption of deformation vibration of methane remained practically unchanged. Starting from the same temperature $\mathrm{T}=160 \mathrm{~K}$ a pressure in the chamber sharply increased and the amplitude of the absorption band at a frequency of observation simultaneously decreased. This process is carried out until complete evaporation of the samples at $\mathrm{T}=$
$180 \mathrm{~K}$. It should be noted that methane contained in the samples throughout the range of temperatures from 160 to $180 \mathrm{~K}$.

\section{Conclusions}

Our studies have shown that in the process of co-condensation of methane and water on the substrate at the temperature of $\mathrm{T}=16 \mathrm{~K}$ a twocomponent solid film was formed. Measuring the vibrational spectra of the samples we have found a little "blue" shift relatively to the spectra of pure solid methane, amounting to the value of the bending vibration of about $14 \mathrm{~cm}^{-1}$ and for $\mathrm{CH}$ stretching vibrations of approximately $5 \mathrm{~cm}^{-1}$. It is virtually identical to the data for nitrogen and argon matrices, from which we can conclude that the state of the methane molecule and its vibrational spectrum are weakly dependent on the composition in the discussed mixtures.

At this stage of research we can make some assumptions relatively the status of methane molecules in the "matrix" of water which are based on the comparison of the thermal desorption curves and of thermograms of amplitude variations of the absorption characteristic vibration frequencies of methane. In our view, it is natural to assume that under these conditions cryoprecipitated methane in solid solution with water can exist in three states. Firstly, it is a condensed state, i.e. solid phase of methane. Secondly, the methane can be in an adsorbed state. The role of absorbent is played by the amorphous solid water (ASW). It is a condition characterized for water cryovacuum condensates formed at $\mathrm{T}=16 \mathrm{~K}[14,15]$. Thirdly, methane may be in a bound state with the molecules of water which are forming clathrates. This, indeed, is the subject of our study. In this paper we attempt to determine the temperature ranges of these states, based on the properties of amorphous solid water ASW and comparing obtained thermograms of desorption and absorption amplitudes of deformation vibrations of methane. As it can be seen in Fig. 6-8 the data for more detailed analysis can be divided into four main temperature ranges.

1.Temperature range from $30 \mathrm{~K}$ to $58 \mathrm{~K}$. In our opinion, the cause of the desorption peak and a sharp change of the amplitude of the absorption frequency of observation $v=1305 \mathrm{~cm}^{-1}$ is the evaporation of solid methane, which was condensed at $\mathrm{T}=$ $16 \mathrm{~K}$. This assumption is consistent with the values of methane equilibrium parameters (pressure, tem- 
perature) on the phase diagram. Desorption peaks at about $\mathrm{T}=20 \mathrm{~K}$, may not relate to the sample and, possibly, are connected with condensates at nonworking surface of the cryostat.

2. Temperature range from $60 \mathrm{~K}$ to $130 \mathrm{~K}$. There is a monotonic decrease in the content of methane adsorbed in the sample, associated with a decrease in the adsorption capacity of the amorphous film of water at higher temperatures. The process is extended in time, so that the desorbed methane pumped from the chamber without the formation of a peak of desorption.

3.Temperature range from $130 \mathrm{~K}$ to $145 \mathrm{~K}$. The observed sharp changes of the measured parameters are related, in our opinion, to the restructuring of the solid phase from the water - amorphous state (ASW) through the intermediate state super cold liquid (SCL) to cubic ice $[4,15]$. It is due to the presence of intermediate SCL-state, adsorbed methane gets necessary diffusion mobility to move to the phase boundary and subsequent desorption.

4. The temperature range of $\mathrm{T}=145 \mathrm{~K}$ before the temperature of evaporation of the sample. In the range from 145 to $160 \mathrm{~K}$ the methane content in the sample is virtually unchanged. Thus the quantity of methane in the samples is sufficiently large. In particular, if we judge by the value of the amplitude of the absorption, the $10 \%$ sample at $160 \mathrm{~K}$ contains at least $60 \%$ of the original content of methane (see Pic. 5). At these high temperatures, methane may be in the film only in the bound state with the water, namely in the form of clathrates. Increase of the temperature above $160 \mathrm{~K}$ leads to a decrease of me- thane content, which is most likely connected with the transition of cubic ice $I_{c}$ to regular hexagonal state $\mathrm{I}_{\mathrm{h}}$, which is apparently accompanied by a partial destruction of the clathrates. Narrow temperature range 172-176 K (Pic. 8) with constant values of the methane content corresponds to the existence of hexagonal ice containing methane clathrates. Further increase of temperature leads to evaporation of the water film with simultaneous change of methane content, i.e. its evaporation. The fact that methane leaves the sample together with the evaporating water confirms presence of methane clathrates in the samples.

Thus, based on experimental studies, we made the following conclusions.

1. A two-component film containing methane clathrates forms in the process of cryovacuum cocondensation of water vapor and methane on the substrate at $\mathrm{T}=16 \mathrm{~K}$.

2. The behavior of the samples during the temperature increase depends on the concentration ratios of water and methane. However, the characteristic temperature ranges are associated with the equilibrium properties of methane and structural phase transformations of amorphous solid water, the variation of the measured parameters for all samples had the same general characteristics.

3 . The samples contain methane until the evaporation of ice at a temperature of about $\mathrm{T}=180 \mathrm{~K}$. This may serve as confirmation of the assumption of the presence of methane clathrates in the studied samples.

\section{References}

[1] Drobyshev A., Aldiyarov A., Katpaeva K., Korshikov E., Kurnosov V., Sokolov D. Transformation of cryovacuum condensates of ethanol near the glass transition temperature // Low Temp. Phys. - 2013. - Vol. 39. - No 8. - P. 714-718.

[2] Aldiyarov A., Drobyshev A., Korshikov E., Kurnosov V. and Sokolov D. On the problem of the existence of a supercooled liquid phase of cryovacuum ethanol condensates // Physics of the Solid State. - 2012. - Vol. 54. - No. 7. - P. 1475-1479.

[3] Jenniskens P., Blake D.F. Crystallization of amorphous water ice in the solar system // Astrophys. J. - 1996. - Vol. 473. - P. 1104-1113.

[4] Aldiyarov A., Aryutkina M., Drobyshev A., Kaikanov M. and Kurnosov V. Investigation of dynamic glass transitions and structure transformations in cryo-vacuum condensates of ethanol // Low Temp. Phys. - 2009. Vol. 35. - No. 4. - P. 251-255.

[5] Talon C., Ramos M., Vieira S., Guello G., Bermejo F., Griado A., Senent M., Bennington S., Fischer H., Schober H. Low-temperature specific heat and glassy dynamics of a polymorphic molecular solid // Physical Review. - 1998. - Vol. 58(2). - P. 745-755.

[6] Fajardo M.E. and Tam S. Observation of the cyclic water hexamer in solid parahydrogen // J. Chem. Phys. 2001. - Vol. 115. - P. 6807-6810.

[7] Tursi A.J. and Nixon E.R. Matrix-Isolation Study of the Water Dimer in Solid Nitrogen // J. Chem. Phys. 1970. - Vol. 52. - P. 1521-1528.

[8] Paul J.B., Collier C.P., Saykally R.J., Sherer J.J. and Keefe A.O. Direct Measurement of Water Cluster Concentrations by Infrared Cavity Ringdown Laser Absorp- 
tion Spectroscopy. J. Phys. Chem. - 1997. - Vol. 101. P. 5211-5214.

[9] Manzhelii V., Freiman Y. Physics of cryocrystals. NY: AIP, Woodbury, 1996. - 691 p.

[10] Debenedetti P.G. Supercooled and glassy water // J. Phys. Cond. Matter. - 2003. - Vol. 15. - P. 1670-1721.

[11] Johari G.P., Hallbrucker A. and Mayer E. Two Calorimetrically Distinct States of Liquid Water Below 150 Kelvin // Science. - 1996. - Vol. 273. - P. 90-92.

[12] Drobyshev A., Aldiyarov A., Zhumagaliuly D., Kurnosov V., Tokmoldin N. Thermal desorption and IR spectrometric investigation of polyamorphic and polymorphic transformations in cryovacuum condensates of water // Low Temp. Phys. - 2007. - Vol. 33(5). - P. 472480.

[13] Drobyshev A., Aldiyarov A., Kurnosov V., Tokmoldin N. Thermally stimulated transformations in cryovacuum water ices // Low Temp. Phys. - 2007. - Vol. 33(4). - P. 355-361.

[14] Johari G.P. Water's Tg-endotherm, sub-Tg peak of glasses and Tg of water // J. Chem. Phys. - 2003. - Vol. 119. - P. 2935- 2937. 